\title{
Reactivity and Binding Energies of Transition Metal Halide Ions with Benzene
}

\author{
Alexei Gapeev and Robert C. Dunbar \\ Chemistry Department, Case Western Reserve University, Cleveland, Ohio, USA
}

We have generated novel halogen-ligated transition metal ions $\mathrm{MX}_{\mathrm{n}}^{+}(\mathrm{M}=\mathrm{Sc}, \mathrm{Ti}, \mathrm{V}$, and $\mathrm{Fe}$, $\mathrm{X}=\mathrm{Cl}, \mathrm{Br}$ and $\mathrm{I}, \mathrm{n}=1-3$ ). We have explored their reactions with benzene, a typical aromatic hydrocarbon. Attachment of one benzene molecule is usually rapid, whereas attachment of a second benzene molecule is generally much slower. The kinetics were analyzed to estimate binding energies, modeling the attachment reaction as a radiative association process. In all cases the Standard Hydrocarbon semiquantitative estimation approach was employed, and in some cases the more accurate variational transition state (VTST) kinetic modeling approach was also applied. Density functional (DFT) quantum calculations were also performed to give computed binding energies for some of the complexes. Taking previously determined binding energies for halogen-ligated alkaline-earth ions as benchmarks, it is concluded that binding of the first benzene molecule to the transition-metal species is strongly enhanced by specific chemical interactions, while binding of the second benzene molecule is more nearly electrostatic. The binding energies are not strongly dependent on the identity of the transition metal ion, and the metal-ion dependences can be rationalized in terms of valence-orbital occupations of the metals. The binding energies are nearly independent of the identity of the halogen ligands. (J Am Soc Mass Spectrom 2002, 13, 477-484) (c) 2002 American Society for Mass Spectrometry

$\mathrm{T}$ The interactions between metal ions and aromatic pi faces of biomolecules have been shown to play an important role in many aspects of the chemistry of living systems, e.g., protein folding, enzyme catalysis, etc. [1]. Cation-pi interactions in biological systems involve metal ions in their stable oxidation state $(+1$ for alkali metals, +2 for alkaline-earth metals, usually +2 and +3 for the transition metals). This means that gas-phase studies of singly charged systems have direct biological relevance for the alkali ions, but have less clear relevance for most other metal ions. However, using double or triply charged gas-phase metal ions in the absence of solvent leveling introduces large electrostatic interactions that are likely to swamp the chemical interactions one would like to study in the gas phase. A way to resolve this dilemma is to attach ligands to the singly charged metal, thereby raising its oxidation state to its biologically relevant value. Halogen ligands seem to be the best choice because they do not migrate from the metal center nor do they promote ion-molecule reactions.

Published online March 27, 2002

This article is presented in honor of Peter Armentrout, whose seminal contributions to ion thermochemistry were recognized with the 2001 Biemann Medal Award.

Address reprint requests to Dr. Robert C. Dunbar, Chemistry Department, Case Western Reserve University, Cleveland, Ohio 44106, USA. E-mail: rcd@po.cwru.edu
Our previous work in pursuit of this strategy [2] investigated the reactions and binding energies of singly charged, halogenated alkaline-earth metal ions with benzene and mesitylene. The binding energies of the halogen-metal ions to benzene were greatly enhanced relative to the bare metal ions, although still much smaller than the predicted binding energies of bare doubly charged ions. It appeared that the binding in these cases was well described as electrostatic in nature, and that the $\mathrm{MX}^{+}$ions showed behavior intermediate between singly-charged and doubly-charged character. A useful description was that the halogenated metal ions behaved toward the benzene ligand with an effective charge between +1 and +2 . For $\mathrm{MgCl}^{+}$a simple classical-electrostatic analysis suggested an effective charge of +1.3 .

More interesting and less predictable are the transition metal ions, which offer a natural extension in this program. We set out to find useful routes to synthesize $\mathrm{MX}_{\mathrm{n}}^{+}$reagent ions in the Fourier-transform ion cyclotron resonance (FT-ICR) cell, and to characterize the reactivity and binding of these ions with benzene. Successful work has been possible with four transition metal ions, whose behavior contrasts in interesting ways with that observed for alkaline earth metal ions.

The gas-phase reactivity of ligated transition metal cations has been an active field for more then two decades. One of the early accounts addressed the gas- 
phase chemistry of $\mathrm{TiCl}_{\mathrm{n}}^{+}$[3]. Later Mandich et al. reported enhanced reactivity of $\mathrm{MCl}^{+}$species $(\mathrm{M}=\mathrm{Mn}$, $\mathrm{Cr}$ and $\mathrm{Fe}$ ) toward small molecules compared to that observed for bare monocations [4].

The thermochemistry of ligated transition metals in the gas phase has been investigated quite thoroughly. Electron diffraction and vibrational spectroscopy studies on gas-phase metal $(\mathrm{Mn}-\mathrm{Co})$ dihalides $(\mathrm{Cl}, \mathrm{Br})$ demonstrated a linear configuration of these species [5]. The Schwarz group has been actively involved in research in the field of gas-phase reactivity, thermochemistry and structure of metal-containing ions that are amenable to mass spectrometry approaches. Recent accounts dealing with ion chemistry of iron chloride and oxide clusters are particularly worth mentioning $[6,7]$.

Addressing the reactivity of bare transition-metal cations and their clusters in the context of the metal center's role in catalysis is an active area of research. For example, bare titanium and titanium dichloride cations are capable of cleaving the aromatic ring of a heterocyclic molecule such as pyridine [8].

Association between aromatic neutrals and transition metal cluster ions has also been described. $\mathrm{FeL}^{+}$ $\left(\mathrm{L}=\mathrm{O}, \mathrm{C}_{4} \mathrm{H}_{6}, \mathrm{c}^{-} \mathrm{C}_{5} \mathrm{H}_{6}, \mathrm{C}_{5} \mathrm{H}_{5}, \mathrm{C}_{6} \mathrm{H}_{6}\right.$ and $\left.\mathrm{C}_{5} \mathrm{H}_{4} \mathrm{CH}_{2}\right)$ form adducts with aromatic heterocycles [9].

\section{Experimental}

All experiments were performed on a modified Nicolet FTMS-2000 spectrometer with a $3 \mathrm{~T}$ superconducting magnet, equipped with a $10 \times 5 \times 5 \mathrm{~cm}$ reaction cell, an IonSpec data system and a Nd-Yag pulsed laser. The setup has been described in detail in our previous work [2]. Benzene was introduced through a leak valve. Ion gauge readings were corrected by the previously known correction coefficient for benzene based on known ion-neutral reaction rate constants. Neutral reactant pressure was typically in the range $3-5 \times 10^{-8}$ torr.

The $\mathrm{MX}_{\mathrm{n}}^{+}$species were prepared by halogen abstraction from the appropriate methyl halide (methyl chloride, methyl bromide or methyl iodide) by singlycharged bare metal cations. The cations were generated by laser ablation-desorption from a metal target. The neutral methyl halide was admitted to the vacuum chamber through a pulsed valve immediately after the laser shot. The pulse duration was optimized to maximize the yield of the desired metal halide ion.

Two different approaches were used to deliver the methyl halides into the vacuum system: For methyl chloride or methyl bromide an evacuated gas bulb was filled with few hundred torr of the corresponding methyl halide from a lecture bottle (Matheson Tri-Gas, Montgomeryville, PA). The gas bulb was then connected to the pulsed-valve inlet. For methyl iodide, $100-500 \mathrm{mg}$ of liquid (Aldrich, Milwaukee, WI) was placed in a $5 \mathrm{ml}$ flask which was attached to the pulsed valve inlet. Air was eliminated by two freeze-pumpthaw cycles.

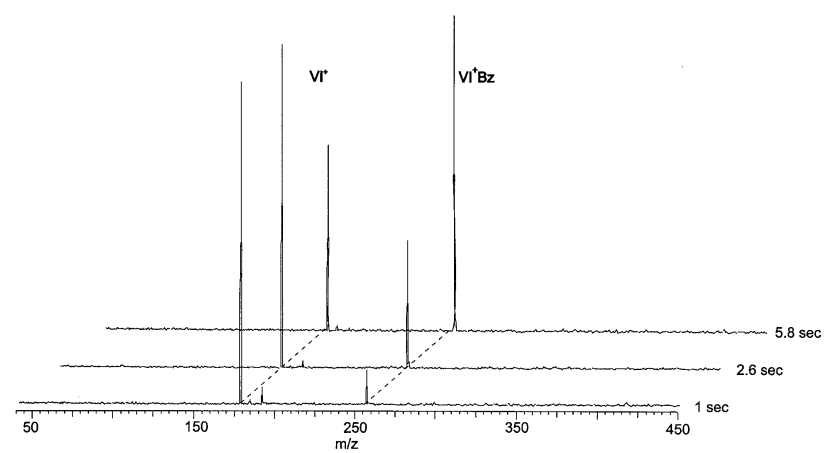

Figure 1. Illustrative spectra showing the formation of $\mathrm{VI}^{+}$ complexes with benzene molecule. Benzene pressure $5 \times 10^{-8}$ torr.

After the pulse of the neutral methyl halide died away, the metal halide ion of interest was isolated by ejection of unwanted ions. The intensities of halide ions and of product ions were monitored by FT-ICR detection as a function of reaction time to derive the association rate constant. A typical series of spectra is shown in Figure 1. In cases where both monobenzene and dibenzene complexes were formed under the given conditions, the successive additions of the first and second benzene ligands to the $\mathrm{MX}_{\mathrm{n}}^{+}$ion were fitted to a sequential pseudo-first-order kinetic scheme, and rate constants were obtained from a least-squares fit. In other cases when there was a substantial difference between first and second benzene molecule attachment rates, and only one of the association processes was important at a given temperature, a simple pseudofirst-order reaction scheme was used, as illustrated for a typical fit in Figure 2. The association rates at early times after the ion-formation sequence were sometimes low, reflecting non-thermalized reactant ions, and it was necessary for the reactant ions to undergo several thermalizing collisions before stable kinetics were observed. These cases were obvious, and it was straightforward to make valid fits by dropping the inconsistent early-time points from the fitted data sets.

There are a number of factors affecting the accuracy

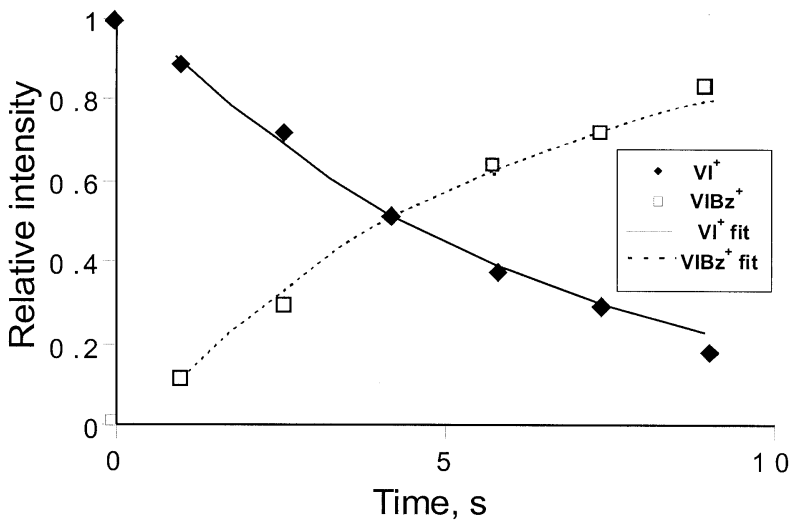

Figure 2. Time plot for the $\mathrm{VI}^{+} /$Benzene system, showing the fit using the rate constant displayed in Table 2. 


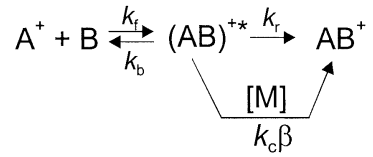

Scheme 1

of the kinetics determination. The largest error is caused by the uncertainty of the benzene pressure measurements, which is estimated to be $30 \%$. Other uncertainties, such as the variation in peak-height ratios with conditions in the ICR cell, gave smaller uncertainties in the derived rate constants. Assuming that the pressure uncertainty dominated over other uncertainties, the rate constants were assigned an absolute error of $\pm 30 \%$. This translates to a $\pm 3 \mathrm{kcal} \mathrm{mol}^{-1}$ contribution to the error in binding energy assignment, which can be assigned as the experimental uncertainty in the binding energy measurements. The actual uncertainty of the derived binding energies is hard to assess, because the accuracy with which binding energies can be derived from association kinetics measurements has not been well established.

Quantum-chemical computations were needed for two purposes. In the first place, vibrational frequencies with their corresponding infrared radiative intensities were used in the VTST kinetic modeling; and in the second place, the quantum-mechanical energies were used to give direct computed binding energies of several complexes. All computations used the densityfunctional (DFT) approach. A recent comparison [10] suggests that the MPW1PW91 functional provides slightly better agreement with experiment for transition-metal benzene complexes than the popular B3LYP functional, and the former functional was employed here. A mixed valence basis set was used with 6-31g(d) on hydrogen, $6-31+\mathrm{g}(\mathrm{d})$ on carbon, and $6-311+\mathrm{g}(\mathrm{d})$ on chlorine and on the metal. As found in other similar studies of our group [12-14], this basis set is sufficient to reduce basis set superposition errors (BSSE) near or below $1 \mathrm{kcal} \mathrm{mol}^{-1}$, which is unimportant compared with other uncertainties.

The computed results were used to estimate absolute binding energies for three systems, $\mathrm{ScClBz}^{+}, \mathrm{TiClBz}^{+}$ and $\mathrm{VClBz}^{+}$. Zero-point energy (ZPE) corrections to these three binding energies were calculated as -0.3 , -1.6 and $-1.8 \mathrm{kcal} \mathrm{mol}^{-1}$, respectively, using the harmonic vibrational frequencies. Corrections for basis set superposition error (BSSE) were calculated as -0.8 , -0.9 and $-0.8 \mathrm{kcal} \mathrm{mol}^{-1}$, respectively, using the geometry-consistent approach of Xantheas [11]. The calculations were carried out with the Gaussian 98 program package [15].

\section{Data Analysis}

In a radiative association reaction two gas-phase species cluster together forming a product ion with photon(s) being emitted (Scheme 1). A collision between the ion A and the molecule $B\left(k_{f}\right)$ gives a metastable ion-molecule complex $(\mathrm{AB})^{+*}$. The stabilization of $\mathrm{AB}^{+*}$ occurs either through a collision with a third body $\left(k_{c} \beta\right)$ or through emission of photon $\left(k_{\mathrm{r}}\right)$. Radiative association experiments like the present ones use a pressure low enough for the $k_{c}$ process to be negligible, and analyze the competition between the $k_{b}$ and $k_{r}$ processes to yield a value for the binding energy of $\mathrm{AB}^{+}[16,17]$.

Two levels of sophistication were employed in the analysis of kinetic data to obtain binding energies from radiative association rate constants. One, the Standard Hydrocarbon Model (SHM) [16] is based on the assumption that all complexes have generic properties. This method provides approximate but useful estimates of binding energy, and is particularly convenient for comparing properties of similar systems.

The other, and more accurate, approach to data analysis makes use of kinetic modeling by Variational Transition State Theory (VTST) [17, 18]. The input required for this model includes vibrational frequencies and infrared emission intensities that are either calculated ab initio or experimentally measured. VTST kinetic modeling as implemented in the VariFlex software package [19] was used to obtain the binding energies for the $\mathrm{ScCl}^{+}, \mathrm{TiCl}^{+}$and $\mathrm{VCl}^{+}$complexes with benzene.

In our recent study of binding alkaline earth halide ions to aromatic hydrocarbons a "diatomic correction" was introduced as an additional parameter for the SHM. This parameter was justified as arising from the restricted rotational phase space of diatomics, and was assigned to improve the agreement between the SHM estimates and the corresponding full VTST calculations for $\mathrm{CaCl}^{+}$and $\mathrm{SrCl}^{+}$. Since the present SHM binding energies were similarly found to be larger than the corresponding VTST-derived values, it seemed appropriate to make a correction for the present systems. Accordingly, in order to bring the SHM values into better agreement with the values derived by full VTST workup, an empirical correction factor of 1.1 was adopted in the present work for all cases involving the attachment of the first benzene to a $\mathrm{MX}_{\mathrm{n}}^{+}$ion. In those cases where comparison is possible against values from full VTST data analysis, or values from quantum chemical energy calculations, the SHM values corrected by this empirical factor appear to be good semiquantitative estimates of the binding energies. The atomic-ion reactants were corrected by the well established atomic-ion correction of 1.4 [16].

The "atomic ion" correction appropriate in applying the SHM to systems with atomic ion reactants, and the possible necessity of extending this to a "diatomic ion" correction for cases with a diatomic-ion reactant, were considered carefully in Refs. [16] and [20]. In Ref. [20] it was found that a correction of the order of a factor of 5-10 to the rate constants (corresponding to a factor of 1.1 or 1.2 for the binding energies) brought the SHM values into good agreement with the VTST values for the $\mathrm{NO}^{+} /$ketone systems. This is encouraging for the application of a factor of 1.1 in the present diatomic-ion 
cases. However, Ref. [20] also raised a cautionary note, pointing out that the assumptions of the SHM (in particular the generic expression used for infrared radiative intensities) can be quite substantially in error for particular classes of systems, and it is necessary to verify the correctness of the SHM for each given set of related systems. Thus, although the plausibility of the present correction factor of 1.1 to the binding energies can be justified by thinking of it as a "diatomic ion" correction, it seems more realistic to consider this as an empirical correction factor to account not only for a diatomic effect, but also for the fact that these $\mathrm{MX}^{+}$ reactant ions and their complexes may differ from the generic hydrocarbon model in their vibrational and radiative properties.

Although more accurate than the SHM, the full VTST data workup in these systems is nevertheless somewhat uncertain. The two bending vibrations of the Cl-M-Bz system (where $\mathrm{Bz}$ represents benzene) have very low frequencies (well below $100 \mathrm{~cm}^{-1}$ ), and the binding energy assignments are quite sensitive to these frequencies. Calculation of these two low-frequency modes is somewhat uncertain, leading to an uncertainty of $\pm 1-2$ $\mathrm{kcal} \mathrm{mol}^{-1}$ associated with this aspect of the kinetic modeling. This uncertainty was particularly severe $( \pm 4$ $\mathrm{kcal} \mathrm{mol}^{-1}$ ) for $\mathrm{VClBz}^{+}$, where these two mode frequencies were very low and method dependent. For instance, B3LYP/DFT in the GAUSSIAN implementation gave two very low real frequencies $\left(16\right.$ and $\left.22 \mathrm{~cm}^{-1}\right)$, while MPW1PW91/DFT gave two imaginary frequencies for the same complex. For this case, a larger uncertainty in the VTST energy assignment was assigned corresponding to the range of vibrational frequencies $\left(40-125 \mathrm{~cm}^{-1}\right)$ that we considered reasonable by analogy with the other, better-behaved metals. $\left(\mathrm{TiClBz}^{+}\right.$, for example, was calculated to have these two frequencies near $60 \mathrm{~cm}^{-1}$ ).

\section{Results}

\section{Transition Metal Halide Formation}

$\mathrm{Sc}^{+}, \mathrm{Ti}^{+}, \mathrm{V}^{+}$and $\mathrm{Fe}^{+}$react with $\mathrm{CH}_{3} \mathrm{X}(\mathrm{X}=\mathrm{Cl}, \mathrm{Br}, \mathrm{I})$, yielding cations containing 1,2 and occasionally 3 halogen atoms. The remainder of the metal cations comprising the first transition row showed no reactivity towards the neutral methyl halides, nor did they react with the other neutral reagents $\left(\mathrm{ICl}\right.$ and $\mathrm{CX}_{4}$ ) that were successfully used in the previous alkaline-earth halide ion study [2]. Attempts at laser desorption/ionization on metal salts $(\mathrm{Ni}, \mathrm{Co}, \mathrm{Mn})$ did not result in any metal halide cations. A promising approach to synthesizing further metal halide ions is that of Mandich et al. [4], who used carbonyl complexes $\mathrm{Cp}(\mathrm{Cr}, \mathrm{Fe})(\mathrm{NO})_{2} \mathrm{Cl}$ to yield $\mathrm{CrCl}^{+}$and $\mathrm{FeCl}^{+}$, but this method was not tried in the present work. Thus the four transition metals described here were the only ones for which we succeeded in synthesizing useful $\mathrm{MX}_{\mathrm{n}}^{+}$reactant ions. Table 1 shows the species that were observed in this study.
Table 1. Number of halogen atoms attached to a metal center

\begin{tabular}{lccc}
\hline & $\mathrm{Cl}$ & $\mathrm{Br}$ & $\mathrm{I}$ \\
\hline \hline $\mathrm{Sc}$ & 1,2 & 1 & 1,2 \\
$\mathrm{Ti}$ & $1,2,3$ & $1,2,3$ & 1,2 \\
$\mathrm{~V}$ & 1 & 1 & 1 \\
$\mathrm{Fe}$ & 1 & 1 & 1,2 \\
\hline
\end{tabular}

\section{Benzene Molecule Attachment}

At room temperature the metal halide ions form 1:1 complexes with benzene at rates approaching the Langevin collisional rate. The kinetic analysis of reactions proceeding near collisional saturation provides only lower limits to the binding energies. Conducting the association reaction at $80^{\circ} \mathrm{C}$ lowered the reaction rates by a factor of 10 and allowed more accurate binding energy values to be determined. Tables 2 and 3 show the rate constants measured for radiative association of metal ions and metal halide ions to benzene, along with binding energies derived from other data analysis approaches and from DFT calculations. Table 4 summarizes results of the present work along with our previous study of alkaline earth metals. Values derived from TCID (threshold collision-induced dissociation) data of Armentrout's group and various computational studies are also summarized for comparison.

All of the transition metal halide cations given in Table 1 attach one molecule of benzene according to the radiative association pathway (Scheme 1), with the following exceptions: (1) $\mathrm{TiBr}_{3}^{+}$forms a substitution product $\mathrm{TiBr}_{2} \mathrm{Bz}^{+}$in competition with the association product; (2) $\mathrm{FeI}_{2}^{+}$reacts with benzene forming $\mathrm{FeIBz}^{+}$; (3) $\mathrm{TiCl}_{3}^{+}$has as its major reaction channel with benzene the charge transfer process producing $\mathrm{TiCl}_{3}+\mathrm{Bz}^{+}$with a rate constant of $3.3 \times 10^{-10} \mathrm{~cm}^{3}$ molec $^{-1} \mathrm{~s}^{-1}$.

The attachment rate constants for a second benzene molecule at a given temperature were usually much lower than those for the first benzene molecule, when they were observed at all. None of the vanadium halide ions attaches a second benzene molecule with an observable rate, nor does any cation containing more than one halogen atom except for $\mathrm{ScCl}_{2}^{+}$.

\section{Discussion}

As a way of measuring binding energies, the radiative association approach is subject to various uncertainties even using the best possible VTST modeling to work up the data. The Standard Hydrocarbon estimation approach used for many of the complexes is still more uncertain. Thus it is important to find as many points of comparison as possible with values from other experimental approaches and those from credible computational studies. Several of the metal-ion monobenzene and dibenzene complexes have been measured by threshold collisional dissociation (TCID) [21], and values derived from these data are included in Table 4.

For the atomic metal ions with benzene, the SHM 
Table 2. Rate constants and binding energies for the radiative association of transition metal inos and their halides with benzene

\begin{tabular}{|c|c|c|c|c|c|}
\hline \multirow[b]{2}{*}{ Ion } & \multirow{2}{*}{$\begin{array}{c}\text { Rate constants } \\
\left(80^{\circ} \mathrm{C}\right), \mathrm{cm}^{3} \mathrm{~s}^{-1} \\
\mathrm{molec}^{-1}\end{array}$} & \multicolumn{4}{|c|}{ Binding energy, kcal mol-1a } \\
\hline & & SHM & $\mathrm{SHM}_{\text {corr }}{ }^{\mathrm{b}}$ & $\begin{array}{c}\text { Best } \\
\text { experiment }^{c}\end{array}$ & DFT \\
\hline$\overline{\mathrm{Sc}^{+}}$ & $\mathrm{H}_{2}$ loss & - & - & - & $48.2^{d}$ \\
\hline $\mathrm{ScCl}^{+}$ & $8.0 \times 10^{-11}$ & 64 & 58 & 53 & 59.8 \\
\hline $\mathrm{ScBr}^{+}$ & $9.0 \times 10^{-11}$ & 66 & 60 & & \\
\hline $\mathrm{ScCl}_{2}{ }^{+}$ & $7.0 \times 10^{-11}$ & 64 & 58 & & \\
\hline $\mathrm{Scl}^{+}$ & $7.8 \times 10^{-11}$ & 65 & 59 & & \\
\hline $\mathrm{Scl}_{2}^{+}$ & $2.6 \times 10^{-11}$ & 60 & 56 & & \\
\hline $\mathrm{Ti}^{+}$ & $1.4 \times 10^{-10}$ & 72 & 51 & 60 & $63.5^{d}$ \\
\hline $\mathrm{TiCl}^{+}$ & $1.6 \times 10^{-10}$ & 69 & 63 & 63 & 66.9 \\
\hline $\mathrm{TiBr}^{+}$ & $1.4 \times 10^{-10}$ & 69 & 63 & & \\
\hline $\mathrm{BiBr}_{2}{ }^{+}$ & $9.0 \times 10^{-11}$ & 66 & 60 & & \\
\hline $\mathrm{TiCl}_{2}{ }^{+}$ & $1.6 \times 10^{-10}$ & 69 & 63 & & \\
\hline $\mathrm{Til}^{+}$ & $1.1 \times 10^{-10}$ & 68 & 62 & & \\
\hline $\mathrm{Til}_{2}^{+}$ & $3.0 \times 10^{-11}$ & 61 & 57 & & \\
\hline $\mathrm{V}^{+}$ & $1.0 \times 10^{-9}$ & & $>55$ & 54 & $50.8^{d}$ \\
\hline $\mathrm{VCl}^{+}$ & $1.9 \times 10^{-10}$ & 71 & 65 & 66 & 72.1 \\
\hline $\mathrm{VBr}^{+}$ & $2.0 \times 10^{-10}$ & 72 & 65 & & \\
\hline $\mathrm{VI}^{+}$ & $1.6 \times 10^{-10}$ & 70 & 64 & & \\
\hline $\mathrm{Fe}^{+}$ & $5.7 \times 10^{-11}$ & 66 & 47 & 50 & $52.4^{d}$ \\
\hline $\mathrm{FeCl}^{+}$ & $2.0 \times 10^{-10}$ & 70 & 64 & & \\
\hline $\mathrm{FeBr}^{+}$ & $2.0 \times 10^{-10}$ & 72 & 65 & & \\
\hline $\mathrm{Fel}^{+}$ & $1.0 \times 10^{-10}$ & 67 & 61 & & \\
\hline
\end{tabular}

aThe uncertainty in binding energies derived from the present data is estimated as $\pm 3 \mathrm{kcal}_{\text {mol }}{ }^{-1}$ due to experimental uncertainties, plus additional uncertainty, which is difficult to assess, arising from the process of converting the experimental rate constants into binding energies via STM or VTST modeling.

bThe corrected SHM values for the atomic-ion reactants include the "atomic-ion correction" of 1.4 multiplying the energy as recommended in Ref. [16]. For the diatomic-ion and triatomic-ion reactants a "linear-ion" correction of a factor of 1.1 was applied as discussed in the text.

${ }^{\mathrm{c}}$ For atomic-ion cases, the values derived from TCID data of Armentrout's group are given [21, 22]. For the diatomic reactants, the values given are from the present work with full VTST analysis. Uncertainties for these latter values are estimated as $\pm 4 \mathrm{kcal}^{\mathrm{mol}}{ }^{-1}$, except for VCIBz ${ }^{+}$, which is estimated as $\pm 6 \mathrm{kcal} \mathrm{mol}^{-1}$.

${ }^{\mathrm{d}}$ Ref. [10].

binding energies derived from radiative association kinetics show inconsistent agreement with accepted values. Agreement for $\mathrm{Ti}^{+}$is poor, whereas agreement for $\mathrm{Fe}^{+}$is good. The rough lower limit imposed by the collisionally saturated $\mathrm{V}^{+}$result is uninformative. As one other example, the binding energy of $\mathrm{Cr}^{+}$derived in earlier work by VTST modeling was higher than accepted values [13]. Thus this approach does not seem to be quantitatively reliable for these transition-metal atomic-ion/benzene systems. On the other hand, for the metal halide ions reacting with benzene, as well as for the reactions involving a second molecule of benzene, the available comparisons indicate reasonable agreement with the radiative association values.

\section{Binding Energy Effects of Varying the Metal and the Halogen}

Figure 1 shows the pattern of binding energy values to a given metal halide ion as a function of the metal ion and of the halogen (corrected SHM modeling), and also shows, for comparison, values for the bare metal cations. It is seen that varying the halogen has virtually no effect on the binding energies for a given metal, even though comparison with the bare metal ion results shows that the presence of a halogen atom increases the binding energy substantially. This might be understood as indicating that the role of the halogen is to oxidize the metal ion (removing one valence electron from the metal's $3 \mathrm{~d} / 4 \mathrm{~s}$ shells), but that it is unimportant which halogen element performs this function.

The only notable difference seen in Figure 3 as a result of varying the metal is that the scandium halide ions bind to benzene slightly less strongly than the other three transition metal halide ions. This observation contrasts with the situation for binding of the bare metal cations with benzene, for which quite large variations are seen across this series of metals. The increase in binding energy on going from $\mathrm{ScCl}^{+}$to $\mathrm{TiCl}^{+}$is easily understood in terms of going from one d electron to two $\mathrm{d}$ electrons, both of which occupy $\mathrm{d}_{\delta}$ orbitals $\left(\mathrm{d}_{\mathrm{xy}}\right.$ and $d_{x^{2-} y^{2}}$ orbitals) which are favorable for $\mathrm{d}_{-} \mathrm{pi}^{*}$ back donation interaction with benzene [23].

Dihalides generally bind to benzene less strongly than the monohalides by a few $\mathrm{kcal} \mathrm{mol}^{-1}$. Although this difference is small for chlorine and bromine cases, the difference between monoiodides and diiodides is substantial (8-10 kcal).

We observed only two species containing more than 2 halogen atoms: $\mathrm{TiCl}_{3}^{+}$and $\mathrm{TiBr}_{3}^{+}$. Neither of them was observed to associate with benzene. The $\mathrm{Ti}$ atoms in these complexes are in a high formal oxidation state of 
Table 3. Rate constants and binding energies for the radiative association of monobenzene complexes with benzene to give the dibenzene complexes

\begin{tabular}{lcc}
\hline lon & $\begin{array}{c}\text { Rate constant } \\
\left(25^{\circ} \mathrm{C}\right) \mathrm{cm}^{3} \mathrm{~s}^{-1} \\
\mathrm{molec}^{-1}\end{array}$ & $\begin{array}{c}\text { SHM binding energy, } \\
\text { kcal mol }\end{array}$ \\
\hline \hline $\mathrm{Sc} \cdot \mathrm{Bz}^{+}$ & & \\
$\mathrm{ScCl} \cdot \mathrm{Bz}^{+}$ & $8.50 \times 10^{-11}$ & 44 \\
$\mathrm{ScBr} \cdot \mathrm{Bz}^{+}$ & $7.40 \times 10^{-11}$ & 43 \\
$\mathrm{ScCl} \cdot \mathrm{Bz}^{+}$ & $1.80 \times 10^{-12}$ & 34 \\
$\mathrm{Scl} \cdot \mathrm{Bz}^{+}$ & $6.02 \times 10^{-11}$ & 43 \\
$\mathrm{Scl} \cdot \cdot \mathrm{Bz}^{+}$ & $2.06 \times 10^{-11}$ & 40 \\
$\mathrm{Ti} \cdot \mathrm{Bz}^{+}$ & & 65 \\
$\mathrm{TiCl} \cdot \mathrm{Bz}^{+}$ & $2.18 \times 10^{-11}$ & 39 \\
$\mathrm{TiBr} \cdot \mathrm{Bz}^{+}$ & $2.12 \times 10^{-11}$ & 39 \\
$\mathrm{TiBr}{ }^{+} \cdot \mathrm{Bz}^{+}$ & $<1 \times 10^{-12}$ & $<30$ \\
$\mathrm{TiCl}{ }_{2} \cdot \mathrm{Bz}^{+}$ & $<1 \times 10^{-12}$ & $<30$ \\
$\mathrm{Til} \cdot \mathrm{Bz}^{+}$ & $2.33 \times 10^{-11}$ & 40 \\
$\mathrm{Til} \cdot \mathrm{Bz}^{+}$ & $<1 \times 10^{-12}$ & $<30$ \\
$\mathrm{~V} \cdot \mathrm{Bz}^{+}$ & & 60 \\
$\mathrm{VCl} \cdot \mathrm{Bz}^{+}$ & $<1 \times 10^{-12}$ & $<30$ \\
$\mathrm{VBr} \cdot \mathrm{Br}^{+}$ & $<1 \times 10^{-12}$ & $<30$ \\
$\mathrm{VI} \cdot \mathrm{Bz}^{+}$ & $<1 \times 10^{-12}$ & $<30$ \\
$\mathrm{Fe} \cdot \mathrm{Bz}^{+}$ & $4.20 \times 10^{-11}$ & 58 \\
$\mathrm{FeCl} \cdot \mathrm{Bz}^{+}$ & $<1 \times 10^{-12}$ & $<30$ \\
$\mathrm{FeBr} \cdot \mathrm{Bz}^{+}$ & $<1 \times 10^{-12}$ & $<30$ \\
$\mathrm{Fel} \cdot \mathrm{Bz}^{+}$ & $<1 \times 10^{-12}$ & $<30$ \\
\hline & &
\end{tabular}

aThe uncertainty in binding energies derived from the present data is estimated as $\pm 3 \mathrm{kcal} \mathrm{mol}^{-1}$ due to experimental uncertainties, plus additional uncertainty, which is difficult to assess, arising from the process of converting the experimental rate constants into binding energies via STM or VTST modeling.

+4 , so that the strong attractive interaction with benzene might make an exothermic channel available.

\section{Comparison with Bare Metal Ions}

For the alkaline earth systems, a large increase in binding energy was found on going from the atomic metal ion to the corresponding halide [2]. It was concluded that this was an electrostatic effect, and in the $\mathrm{MgCl}^{+}$case point-charge modeling suggested that the metal ion has an effective charge of +1.3 . A similar

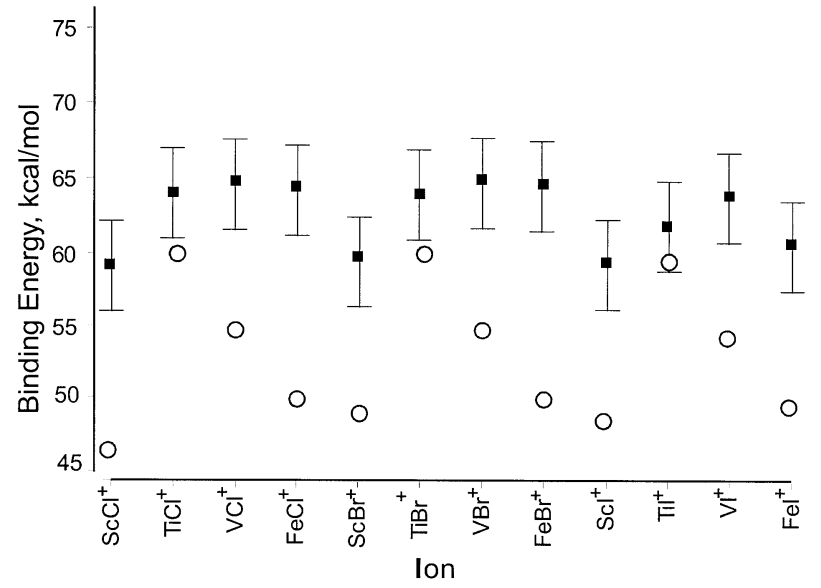

Figure 3. Comparison between $\mathrm{MX}^{+}$affinity to benzene (squares) and $\mathrm{M}^{+}$affinity to benzene (circles). $\mathrm{M}^{+}$values from Ref. [21] except $\mathrm{Sc}^{+}$(Refs. [10, 23]). $\mathrm{MX}^{+}$binding energies are the corrected SHM values from Table 2.

electrostatic effect is expected to operate for the transition metal ion systems. However, superimposed on this are the binding energy effects arising from valence electron interactions of the metal with the benzene ligand. These orbital interactions are strongly metaldependent, in contrast to the electrostatic effects. Furthermore, the orbital effects are drastically different for $\mathrm{M}^{+}$and $\mathrm{MX}^{+}$, because the metal center in $\mathrm{MX}^{+}$has one less valence electron than the bare metal ion. Thus in thinking about orbital effects, it is most useful to think of $\mathrm{TiX}^{+}\left(\mathrm{d}^{2}\right)$ as comparable to $\mathrm{Sc}^{+}\left(\right.$also $\left.\mathrm{d}^{2}\right)$, and $\mathrm{VX}^{+}$ $\left(d^{3}\right)$ as comparable to $\mathrm{Ti}^{+}\left(\mathrm{d}^{3}\right)$.

Looking at Table 4 or Figure 3 for the comparison of $\mathrm{M}^{+}$and $\mathrm{MCl}^{+}$binding energies, the operation of these two effects is clearly seen. In the case of $\mathrm{Ti}$, there is little difference between $\mathrm{Ti}^{+}$and $\mathrm{TiCl}^{+}$. This can be seen as a cancellation of effects. The electrostatic effect, as with the alkaline earths, should give a large binding energy increase. However, the loss of a bonding $d$ electron in going from $\mathrm{d}^{3}$ to $\mathrm{d}^{2}$ is costly in binding energy, as is seen in the fact that going from $\mathrm{Ti}^{+}$to $\mathrm{Sc}^{+}$costs about 15

Table 4. Binding energies of mono- and dibenzene complexes $\left(\mathrm{kcal} \mathrm{mol}^{-1}\right)^{\mathrm{a}}$

\begin{tabular}{|c|c|c|c|c|c|c|}
\hline & $\mathrm{M} \cdot \mathrm{Bz}^{+}$ & $\mathrm{MCl} \cdot \mathrm{Bz}^{+}$ & $\mathrm{MBr} \cdot \mathrm{Bz}^{+}$ & $\mathrm{MBz} \cdot \mathrm{Bz}^{+}$ & $\mathrm{MClBz} \cdot \mathrm{Bz}^{+}$ & $\mathrm{MBrBz} \cdot \mathrm{Bz}^{+}$ \\
\hline $\mathrm{Mg}^{\mathrm{b}}$ & $37(30-32)^{c}$ & 62 & & $<32$ & $<32$ & \\
\hline $\mathrm{Ca}{ }^{\mathrm{b}}$ & 32 & 44 & 39 & 30 & 36 & 32 \\
\hline $\mathrm{Sr}^{\mathrm{b}}$ & 28 & 37 & 37 & 28 & 36 & 30 \\
\hline Sc & $(44-48)^{d}$ & $52^{f}(60)^{g}$ & 60 & & 44 & 43 \\
\hline $\mathrm{Ti}$ & $51(56-63)^{\mathrm{e}}$ & $63^{f}(67)^{g}$ & 63 & $65(61)^{\mathrm{h}}$ & 39 & 39 \\
\hline V & $>55(49-54)^{e}$ & $66^{f}(72)^{g}$ & 65 & $60(59)^{\mathrm{h}}$ & $<30$ & $<30$ \\
\hline $\mathrm{Fe}$ & $47(49-57)^{\mathrm{e}}$ & 64 & 65 & $58(45)^{h}$ & $<30$ & $<30$ \\
\hline
\end{tabular}

${ }^{a}$ Values not otherwise referenced are the values from the present work derived from experimental radiative association measurements by SHM modeling.

${ }^{b}$ Alkaline earth values not otherwise referenced are from Ref. [2].

cValues from quantum calculations and from analysis of TCID experimental data from Refs. [2, 24, 25].

${ }^{\mathrm{d}}$ Calculated values from Refs. [10, 23].

eValues from quantum calculations and from analysis of experimental data from Refs. [10, 21, 22, 23, 26].

fPresent data fitted by VTST modeling.

gPresent DFT/MPW1PW91 calculations.

${ }^{\mathrm{h}}$ TCID value from Ref. [21]. 
$\mathrm{kcal} \mathrm{mol}^{-1}$ of binding energy. The orbital effect in going from $\mathrm{Ti}^{+}$to $\mathrm{TiCl}^{+}$is similar, and would be expected to decrease the binding by perhaps $15 \mathrm{kcal} \mathrm{mol}^{-1}$. This decrease compensates the electrostatic increase, given the net result of not-very-different binding energies for $\mathrm{Ti}^{+}$and $\mathrm{TiCl}^{+}$.

Comparison of $\mathrm{V}^{+}$and $\mathrm{VCl}^{+}$, shows a more substantial increase in binding energy, on the order of $15 \mathrm{kcal}$ $\mathrm{mol}^{-1}$. In this case, the orbital effect is small, as is shown by the comparison between $\mathrm{V}^{+}$and $\mathrm{Ti}^{+}$. The fourth $\mathrm{d}$ electron of $\mathrm{V}^{+}$occupies a d orbital which is unfavorable to benzene bonding, and removal of this electron to go from $\mathrm{V}^{+}\left(\mathrm{d}^{4}\right)$ to $\mathrm{Ti}^{+}\left(\mathrm{d}^{3}\right)$ gives a modest increase of the benzene binding energy. Thus the orbital effect in going from $\mathrm{V}^{+}$to $\mathrm{VCl}^{+}$should give a slight increase. The electrostatic effect in this case then leads to the observed substantial increase in binding energy.

Comparison of $\mathrm{Fe}^{+}$and $\mathrm{FeCl}^{+}$, showing a substantial binding energy increase, is complex to interpret because of the uncertainty and ambiguity of the spin and configuration changes involved in the complexation of iron ions with benzene $[22,23]$. The same argument by analogy may not be valid here, because the loss of bonding d electrons in going from $\mathrm{Fe}^{+} \mathrm{Bz}\left(\mathrm{d}^{7}\right)$ to $\mathrm{Mn}^{+} \mathrm{Bz}$ $\left(d^{5} s\right)$ involves promotion of a d electron to the $4 \mathrm{~s}$ orbital [23] which may not be analogous to the $\mathrm{FeClBz}^{+}$ system.

The DFT calculations describe the $\mathrm{MClBz}^{+}$complexes for $\mathrm{Sc}$ and $\mathrm{Ti}$ as pure d-electron complexes. For the Sc complex, which is $\mathrm{d}^{1}$, the occupied d-orbital is one of the $d_{\delta}$-derived orbitals $\left(d_{x y}\right.$ or $\left.d_{x^{2}-y^{2}}\right)$ which put the electron density in the plane perpendicular to the $\mathrm{Cl}-\mathrm{M}-\mathrm{Bz}$ axis, and minimize repulsion with the ligand electrons. For the Ti complex, which is $d^{2}$, both $d_{\delta^{-}}$ derived orbitals are occupied, again minimizing ligand repulsion. The binding energies of these two complexes may be decreased by a promotion-energy effect, since the highest occupied orbitals in both $\mathrm{ScCl}^{+}$and $\mathrm{TiCl}^{+}$ are calculated to be hybrid $3 \mathrm{~d}-4 \mathrm{~s}$ orbitals with substantial $4 \mathrm{~s}$ character, so that in forming the complex it may be necessary to supply some 4s-to-3d promotion energy. In the vanadium case, with three $3 \mathrm{~d} / 4 \mathrm{~s}$ electrons, both of the $d_{\delta}$-derived orbitals are occupied in both $\mathrm{VCl}^{+}$and $\mathrm{VClBz}^{+}$. The third electron in $\mathrm{VCl}^{+}$occupies a $\mathrm{d}_{\pi}$-derived orbital which mixes with the chlorine pi system (indicating $\mathrm{d}-\mathrm{pi}^{*}$ donation) while for $\mathrm{VClBz}^{+}$the third electron occupies a $3 \mathrm{~d}_{\sigma}-4 \mathrm{~s}$ hybrid orbital which places the electron density largely in the plane perpendicular to the Cl-M-Bz axis. It is unclear from the DFT calculations just how much promotion energy might be involved in forming the $\mathrm{VClBz}^{+}$complex.

\section{Comparison with Alkaline-Earth Metal Halides}

Alkaline-earth metal ions do not readily form halides containing more than one halogen ligand because the metal center in the monohalides is already in a formal oxidation state of +2 , corresponding to complete removal of their valence electrons. Because transition metals have higher oxidation states available, they are able to accommodate more than one halogen while preserving an overall charge of +1 for the halogenide.

Transition metal monohalides bind benzene 20-30 kcal more strongly then the alkaline-earth monohalides (Table 4). As one example, $\mathrm{Ca}^{+}$and $\mathrm{Sc}^{+}$in the halides have comparable sizes $\left(\mathrm{CaCl}^{+}\right.$is $2.31 \AA \mathrm{AcCl}^{+}$is 2.21 $\AA$ ), but the binding energies of their halides to benzene are very different. Comparison of Mulliken charges on Sc and Ca shows no significant difference. This encourages us to think there is a significant covalent character of transition metal halides binding to benzene. The only exception is $\mathrm{MgCl}^{+}$. This ion binds to benzene almost as strongly as the transition metal chlorides. The reason for the exceptionally strong binding in this case may be the fact that the magnesium cation is considerably smaller than the calcium and transition metal cations.

\section{Bis-benzene Complexes}

The SHM binding energy for attachment of a second benzene molecule in transition metal halide/benzene complexes is significantly smaller, on the order of 15-30 $\mathrm{kcal} \mathrm{mol}^{-1}$, than for the first benzene molecule (Table 3 ). This is in contrast to the previously studied alkalineearth metal halides where the second benzene binds 8-15 kcal less strongly than the first. Attachment of a second benzene molecule to the transition metal $\mathrm{MX}_{\mathrm{n}} \mathrm{Bz}^{+}$complexes actually displays energies in the same general range as those found for the alkaline earth cases (see Table 4). We have already established that binding in alkaline-earth metal-halide/benzene complexes is primarily electrostatic. Therefore, weak attachment of a second benzene molecule in the transition metal systems may be an indication of primarily electrostatic interaction between the metal center and the second benzene in all cases. An attractive point of view is that the first benzene ligand takes full advantage of the available possibilities for specific chemical bonding with the transition metal center, and the second ligand is relegated to a position where only electrostatic bonding remains available to it, just as in the alkaline earth systems.

\section{Conclusions}

The transition metal species show an enhancement of binding to the first benzene expected from d-orbital interactions with the ligand, where the benchmark for comparison is the alkaline-earth counterparts. Somewhat unexpected is the observation that this enhancement does not appear to extend to the binding of a second benzene molecule, which appears to be largely electrostatic. The periodic dependence of the binding energies on the identity of the metal can be rationalized to some extent by analogy with the bare metal ions having the same numbers of valence $d$ electrons. The lack of success in synthesizing halogenated reagent ions of a wider variety of transition metals precluded mak- 
ing a comprehensive periodic study of the electronic bonding effects. Such studies await either more versatile experimental approaches to reagent ion synthesis, or more extensive study by quantum chemical calculations.

The insubstantial changes observed in binding energies when one halogen is replaced with another suggest that the role of the halogen ligands is largely confined to oxidizing the metal center, and that one halogen element is not especially more effective than another in this regard. It is interesting that the dihalogenated reagent ions are kinetically similar to the monohalogenated ions in binding the first molecule of benzene, although attachment of the second benzene molecule of benzene is disfavored by the presence of two halogen ligands. A greater steric effect than is indicated by these results might have been expected in going from one to two halogen ligands on the reagent ion, an effect worth further consideration.

\section{Acknowledgments}

The support of the donors of the Petroleum Research Fund, administered by the American Chemical Society, is gratefully acknowledged.

\section{References}

1. Ma, J. C.; Dougherty, D. A. The Cation-Pi Interaction. Chem. Rev. 1997, 97, 1303-1324.

2. Gapeev, A.; Dunbar, R. C. Binding of Alkaline Earth Halide Ions to Benzene and Mesitylene. J. Phys. Chem. A 2000, 104, 4084.

3. Allison, J.; Ridge, D. P. Ion-molecule reactions in mixtures of $\mathrm{TiCl}_{4}$ with olefins in gas phase. J. Am. Chem. Soc. 1977, 99, 35.

4. Mandich, M. L.; Steigerwald, M. L.; Reents, W. D., Jr. The Effect of Chloro Substiution on the Electronic Structures of $\mathrm{ClCr}^{+}, \mathrm{ClMn}^{+}$and $\mathrm{ClFe}^{+}$and Their Reactivity with Small Alkanes. J. Am. Chem. Soc. 1986, 108, 6197-6202.

5. Hargittai, M.; Subbotina, N. Y.; Kolontis, M. Molecular Structure of first row transitional metal dihalides form combined electron-diffraction and vibration spectroscopic analysis. J. Chem. Phys. 1991, 94, 7278-7286.

6. Schröder, D.; Bärsch, S.; Schwarz, H. Redox properties of charged and neutral iron chlorides $\mathrm{FeCl}_{\mathrm{m}}^{\mathrm{n}}(\mathrm{m}=1-3 ; \mathrm{n}=-1,0$, +1, and +2). Int. J. Mass Spectrom. 1999, 192, 125-139.

7. Jackson, P.; Harvey, J. N.; Schröder, D.; Schwarz, H. Structure and reactivity of the prototype iron-oxide cluster $\mathrm{Fe}_{2} \mathrm{O}_{2}^{+}$. Int. J. Mass Spectrom. 2001, 204, 233-245.

8. Wong, P. S.; Ma, S. G.; Cooks, R. G. Ion/molecule reactions of titanium and titanium dichloride cations with pyridine: Activation of the pyridine ring in the gas phase. Rapid Commun. Mass Spectrom. 1996, 10, 927-931.

9. Bakhtiar, R.; Jacobson, D. B. Transition-metal mediated heteroatom removal by reactions of $\mathrm{FeL}^{+}[\mathrm{L}=\mathrm{O}, \mathrm{C} 4 \mathrm{H} 6, \mathrm{c}-\mathrm{C} 5 \mathrm{H} 6$, c-C5H5, $\mathrm{C} 6 \mathrm{H} 6, \mathrm{C} 5 \mathrm{H} 4=\mathrm{CH} 2]$ with furan, thiophene, and pyrrole in the gas phase. J. Am. Soc. Mass Spectrom. 1996, 7, 938-952.

10. Dunbar, R. C.Metal Cation Binding to Phenol: DFT Comparison of the Competing Sites. J. Phys. Chem. 2002, Submitted.
11. Xantheas, S. S. On the Importance of the Fragment Relaxation Energy Terms in the Estimation of the Basis Set Superposition Error Correction to the Intermolecular Interaction Energy. J. Chem. Phys. 1996, 104, 8821-8824.

12. Gapeev, A.; Yang, C.-N.; Klippenstein, S. J.; Dunbar, R. C. Binding Energies of Gas-Phase Metal Ions with Pyrrole: Experimental and Quantum Chemical Results. J. Phys. Chem. A 2000, 104, 3246-3256.

13. Ryzhov, V.; Yang, C.-N.; Klippenstein, S. J.; Dunbar, R. C. Binding Energies of Chromium Cations with Fluorobenzenes from Radiative Association Kinetics. Int. J. Mass Spectrom. 1998, 185/186/187, 913-923.

14. Dunbar, R. C. Complexation of $\mathrm{Na}^{+}$and $\mathrm{K}^{+}$to Aromatic Amino Acids: A Density Functional Computational Study of Cation-p Interactions. J. Phys. Chem. A 2000, 104, 8067-8074.

15. Frisch, M. J.; Trucks, G. W.; Schlegel, H. B.; Scuseria, G. E.; Robb, M. A.; Cheeseman, J. R.; Zakrzewski, V. G.; Montgomery, J. A., Jr.; Stratmann, R. E.; Burant, J. C.; Dapprich, S.; Millam, J. M.; Daniels, A. D.; Kudin, K. N.; Strain, M. C.; Farkas, O.; Tomasi, J.; Barone, V.; Cossi, M.; Cammi, R.; Mennucci, B.; Pomelli, C.; Adamo, C.; Clifford, S.; Ochterski, J.; Petersson, G. A.; Ayala, P. Y.; Cui, Q.; Morokuma, K.; Malick, D. K.; Rabuck, A. D.; Raghavachari, K.; Foresman, J. B.; Cioslowski, J.; Ortiz, J. V.; Stefanov, B. B.; Liu, G.; Liashenko, A.; Piskorz, P.; Komaromi, I.; Gomperts, R.; Martin, R. L.; Fox, D. J.; Keith, T.; Al-Laham, M. A.; Peng, C. Y.; Nanayakkara, A.; Gonzalez, C.; Challacombe, M.; Gill, P. M. W.; Johnson, B.; Chen, W.; Wong, M. W.; Andres, J. L.; Gonzalez, C.; HeadGordon, M.; Replogle, E. S.; Pople, J. A. GAUSSIAN 98, Revision A.6.. Gaussian, Inc.: Pittsburgh, PA, 1998.

16. Dunbar, R. C. Modeling Radiative Association Kinetics. Int. J. Mass Spectrom. Ion Proc. 1997, 160, 1-16.

17. Klippenstein, S. J.; Yang, Y.-C.; Ryzhov, V.; Dunbar, R. C. Theory and Modeling of Ion-Molecule Radiative Association Kinetics. J. Chem. Phys. 1996, 104, 4502-4516.

18. Ryzhov, V.; Yang, Y.-C.; Klippenstein, S. J.; Dunbar, R. C. Temperature Dependence of Radiative Association Rates. J. Phys. Chem. A 1998, 102, 8865-8870.

19. VariFlex computer code, available at http://chemistry.anl.gov/chem-dyn/VariFlex/.

20. Ryzhov, V.; Dunbar, R. C. Size Dependence of Radiative Association Rates. Int. J. Mass Spectrom. Ion Proc. 1997, 167/168, 627-635.

21. Meyer, F.; Khan, F. A.; Armentrout, P. B. Thermochemistry of Transition-Metal Benzene Complexes - Binding Energies of $\mathrm{M}\left(\mathrm{C}_{6} \mathrm{H}_{6}\right)(\mathrm{X})^{+}(\mathrm{X}=1,2)$ for $\mathrm{M}=\mathrm{Ti}$ to $\mathrm{Cu}$. J. Am. Chem. Soc. 1995, 117, 9740-9748.

22. Yang, C.-N.; Klippenstein, S. J. Theory and Modeling of the Binding in Cationic Transition Metal-Benzene Complexes. J. Phys. Chem. A 1999, 103, 1094-1103.

23. Bauschlicher, C. W., Jr.; Partridge, H.; Langhoff, S. R. Theoretical Study of Transition-Metal Ions Bound to Benzene. J. Phys. Chem. 1992, 96, 3273-3278.

24. Andersen, A.; Muntean, F.; Walter, D.; Rue, C.; Armentrout, P. B. Collision-Induced Dissociation and Theoretical Studies of $\mathrm{Mg}^{+}$Complexes with $\mathrm{CO}, \mathrm{CO}_{2}, \mathrm{NH}_{3}, \mathrm{CH}_{4}, \mathrm{CH}_{3} \mathrm{OH}$, and $\mathrm{C}_{6} \mathrm{H}_{6}$. J. Phys. Chem. A 2000, 104, 692-705.

25. Bauschlicher, C. W., Jr.; Partridge, $\mathrm{H}$. $\mathrm{Mg}^{+}$-Ligand Binding Energies. Chem. Phys. Lett. 1991, 181, 129-133.

26. Klippenstein, S. J.; Yang, C. N. Density Functional Theory Predictions for the Binding of Transition Metal Cations to Pi Systems: From Acetylene to Coronene and Tribenzocyclyne. Int. J. Mass Spectrom. 2000, 201, 253-267. 